\title{
Persistently Expansive Geodesic Flows
}

\author{
Rafael O. Ruggiero \\ Pontificia Universidade Católica do Rio de Janeiro, Rua Marqués de São Vicente 255, \\ Gávea, Rio de Janeiro, Brasil
}

Received September 18, 1990; in revised form November 9, 1990

\begin{abstract}
We prove that $C^{1}$-persistently expansive geodesic flows of compact, boundaryless Riemannian manifolds have the property that the closure of the set of closed orbits is a hyperbolic set. In the case of compact surfaces we deduce that the geodesic flow is $C^{1}$-persistently expansive if and only if it is an Anosov flow.
\end{abstract}

\section{Introduction}

In this paper we present some results concerning geodesic flows possessing certain topological properties which persist under small perturbations. Recall that if $(M, g)$ is a complete Riemannian manifold and $T_{1} M$ is its unit tangent bundle, the geodesic flow $\varphi_{t}: T_{1} M \rightarrow T_{1} M$ is defined as follows: given a point $(p, v) \in T_{1} M, \varphi_{t}(p, v)=\left(\gamma(t), \gamma^{\prime}(t)\right)$, where $\gamma(t)$ is the unit geodesic of $M$ such that $\gamma(0)=p$ and $\gamma^{\prime}(0)=v$. Let us denote as $\kappa^{k}(M)$ the set of geodesic flows of Riemannian metrics of $M$ endowed with the $C^{k}$ topology. Given any one parameter family of homeomorphisms $\psi_{t}: N \rightarrow N$ acting on a metric space $N$, we say that it is expansive if there is an $\varepsilon>0$ such that every $p \in N$ satisfies the following property: if $q \in N$ and there exists a continuous surjection $f_{q}: \mathbb{R} \rightarrow \mathbb{R}$ with $d\left(\psi_{t}(p), \psi_{f_{q}(t)}(q)\right) \leqq \varepsilon$ for every $t \in \mathbb{R}$, then there exists $t_{0} \in \mathbb{R}$ depending on $\varepsilon, p$, q, with $t_{0} \rightarrow 0$ if $d(p, q) \rightarrow 0$ such that $q=\psi_{t_{0}}(p)$. When $t \in \mathbb{Z}$ for every $t$ we just take $f_{q}(t)=t$ and $t_{0}=0$.

The persistence of expansivity is closely related with hyperbolicity and stability of dynamical systems. Let $E^{k}(M)$ be the subset of $\kappa^{k}(M)$ of expansive geodesic flows. An Anosov geodesic flow of a compact manifold $M$ is expansive, and since it is $C^{1}$-structurally stable [1] it belongs to $\operatorname{int}\left(E^{1}(M)\right)$ - the interior of $E^{1}(M)$ in $\kappa^{1}(M)$. Axiom $A$ systems are expansive near the closure of the set of periodic orbits, and since they are $\Omega$-stable [10,12] this property persists under $C^{1}$ perturbations. On the other hand, Mañe [6] proves that the interior of the set of expansive diffeomorphisms in $\operatorname{Diff}^{1}(M)$ (i.e. the set of $C^{\infty}$ diffeomorphisms of $M$ endowed with the $C^{1}$ topology) coincides with the 
set of Quasi-Anosov ones. (A diffeomorphism $f: M \rightarrow M$ is called QuasiAnosov if for every $p \in M$ and $V \in T_{p} M$ we have that $\left\|D f^{n}(V)\right\| \mapsto \infty$ when either $n \nrightarrow+\infty$ or $n \nrightarrow-\infty$.) Mañe shows in particular that such diffeomorphisms are Axiom $A$ systems. We obtain in this work analogous results for persistently expansive geodesic flows. If $\psi_{t}$ is a flow acting on a Riemannian manifold $N$ we say that an invariant set $X \subseteq N$ is hyperbolic if there exist constants $C>0,0<\lambda<1$, and a splitting $E_{p}^{s} \oplus E_{p}^{u} \oplus E_{p}=T_{p} N$ for every $p \in X$ such that

i) $E_{p}^{s}, E_{p}^{u}$ are invariant by $d \psi_{t}$ and $E_{p}$ is the direction of the flow at $p$. (If $p$ is a singularity take $E_{p}=0$.)

ii) $\left\|\left.d \psi_{t}\right|_{E_{p}^{s}}\right\| \leqq C \lambda^{t} \forall t \geqq 0$.

$\left\|\left.d \psi_{t}\right|_{E_{p}^{u}} ^{u}\right\| \leqq C \lambda^{-t} \forall t \leqq 0$.

When $X=N$ the flow is called an Anosov flow. Denote as $A(M)$ the set of Anosov geodesic flows of the manifold $M$.

Theorem A. Let $M$ be a compact manifold of dimension two. Then $\operatorname{int}\left(E^{1}(M)\right)$ $=A(M)$.

This theorem will follow from the fact that periodic orbits of expansive geodesic flows of surfaces are dense (see Sect. 3) together with the following result:

Theorem B. Let $(M, g)$ be a compact Riemannian manifold of dimension $n$. If the geodesic flow $\varphi_{t}$ belongs to int $\left(E^{1}\right)$ the set $\overline{P(\varphi)}$ - the closure of the set $P(\varphi)$ of periodic orbits of $\varphi_{t}$ - is a hyperbolic set.

The method used to prove Theorem B combines some classical results concerning symplectic dynamics - the so-called Birkhoff-Lewis fixed point theorem - with the general theory of persistent invariant bundles. Indeed, we deduce that if $\varphi_{t} \in E^{1}(M)$ then the closed orbits are $C^{1}$-persistently hyperbolic, and from [6] this implies that there exists an extended, continuous invariant bundle defined in $\overline{P(\varphi)}$ - which coincides with the hyperbolic splitting along each periodic orbit-satisfying what is called the domination condition (see [6] and also Sect. 2). It is important to remark that the absence of a closing lemma for geodesic flows determines essential differences between the arguments used here and those of [6] for diffeomorphisms. Roughly speaking, Pugh's closing lemma [9] says that for every diffeomorphism $f: M \rightarrow M$ defined on a compact manifold $M$ we can approximate "almost" periodic parts of orbits of $f$ by periodic orbits of $C^{1}$ perturbations of $f$.

On the other hand, we shall show that the splitting mentioned above satisfies an algebraic property associated to the symplectic structure of the geodesic flow: it comes to be a Lagrangian splitting. Invariant splittings of Anosov geodesic flows are easily seen to be Lagrangian, while the reciprocal statement is not necessarily true. What we prove is that a continuous, Lagrangian, invariant splitting defined on a compact invariant set for the geodesic flow is hyperbolic if and only if it satisfies the domination condition. 


\section{Generic Properties of Poincaré Maps of Closed Orbits}

Let $(M, g)$ be a complete Riemannian manifold, and let $T_{1} M$ be unit tangent bundle. The metric $g$ induces a Riemannian structure on $T_{1} M$ given by the metric $\hat{g}$, which we define as follows: Let $\pi: T M \rightarrow M$ be the projection $\pi(p, v)=p$, where $(p, v)$ is a point of $T M$ in local coordinates $U \times \mathbb{R}^{n}, U$ an open subset of $M$. Let

$$
\begin{aligned}
& K: T(T M) \rightarrow T M \\
& K: Y_{(q, v)} \mapsto \bar{V}_{\overline{d \pi(Y)_{q}}} \overline{d \pi(Y)_{q}},
\end{aligned}
$$

where $\bar{V}: T M \times T M \rightarrow T M$ is the Levi-Civita connection of $(M, g)$, and $\overline{d \pi(Y)_{q}}$ is any differentiable vector field defined in an open neighborhood of $p$ such that $\overline{d \pi(Y)_{p}}=d \pi(Y)_{p}$. Then, if $V, W \in T_{\xi}(T M)$, define

$$
\hat{g}(V, W)=g(d \pi(V), d \pi(W))+g(K(V), K(W)) .
$$

It is easy to see that $\operatorname{Ker}\left(K_{\xi}\right) \oplus \operatorname{Ker}\left(d \pi_{\xi}\right)=T_{\xi}(T M)$, where the sum is orthogonal with respect to $\hat{g}$, and $\operatorname{dim}\left[\operatorname{Ker}\left(K_{\xi}\right)\right]=\operatorname{dim}\left[\operatorname{Ker}\left(d \pi_{\xi}\right)\right]=n$. Now, let us consider the restriction of this metric to $T_{1} M$, which we still denote as $\hat{g}$.

Let $\varphi_{t}: T_{1} M \rightarrow T_{1} M$ be the geodesic flow of $(M, g)$. Consider $N_{\xi}=$ $\left\{v \in T_{\xi}\left(T_{1} M\right) \backslash \hat{g}\left(v, E_{\xi}\right)=0\right\}$, where $E_{\xi}$ is the direction tangent to the flow at $\xi \in T_{1} M$, and let $H_{\xi} \subset N_{\xi}, V_{\xi} \subset N_{\xi}$ be the horizontal and the vertical subspaces respectively, where

$$
\begin{aligned}
H_{\xi} & =\operatorname{Ker}\left(K_{\xi}\right) \cap N_{\xi}, \\
V_{\xi} & =\operatorname{Ker}\left(d \pi_{\xi}\right) \cap N_{\xi} .
\end{aligned}
$$

Note that $\operatorname{dim}\left(H_{\xi}\right)=\operatorname{dim}\left(V_{\xi}\right)=n-1$ and $N_{\xi}=H_{\xi} \oplus V_{\xi}$. Define

$$
\begin{aligned}
\mathfrak{I}: T\left(T_{1} M\right) & \rightarrow T\left(T_{1} M\right), \\
\mathfrak{I}(X, Y) & =(-Y, X) .
\end{aligned}
$$

Observe that $\mathfrak{J}^{2}=-I$, and that there exists a canonical symplectic structure on $N_{\xi}$ induced by $J$. Recall that a symplectic form $\omega$ on $\mathbb{R}^{2 m}$ is an alternate, non-degenerate two-form on $\mathbb{R}^{2 m}$. We call the pair $\left(\mathbb{R}^{2 m}, \omega\right)$ a symplectic structure. Now, for each $\xi \in T_{1} M$ consider the following form:

$$
\Omega_{\xi}(V, W)=\hat{g}(V, \mathfrak{I}(W)) \forall V, \quad W \in N_{\xi} .
$$

It is clear that $\Omega_{\xi}$ depends differentiably on $\xi$, and since $\mathfrak{J}$ is both an isomorphism and an involution we get that $\Omega_{\xi}$ is in fact a symplectic form $\forall \xi$. We shall denote as $\Omega$ the two-form in $\Lambda^{2}\left(T_{1} M\right)$, the space of two-forms of $T_{1} M$, defined by $\Omega(\xi)=\Omega_{\xi}$.

A simple, but important remark is that the geodesic flow of $(M, g)$ preserves both $N_{\xi}$ and $\Omega$, i.e., $d \varphi_{t}\left(N_{\xi}\right)=N_{\varphi_{t}(\xi)} \forall \xi \in T_{1} M$ and

$$
\varphi_{t}^{*}\left(\Omega_{\varphi_{t}(\xi)}\right)=\Omega_{\xi} \forall t \in \mathbb{R},
$$

where the map $F^{*}: \Lambda^{2}\left(T_{F(p)} X\right) \rightarrow \Lambda^{2}\left(T_{p} X\right)$ is defined as

$$
F^{*} \omega_{F(p)}(V, W)=\omega_{p}\left(d F^{-1}(V), d F^{-1}(W)\right)
$$


for every diffeomorphism $F: X \rightarrow X$ on a manifold $X$, with $\Lambda^{k}\left(T_{p} X\right)$ being the space of $k$ forms on $T_{p} X$. This implies in particular that $\hat{g}\left(d \varphi_{t}(V)\right.$, and $\left.\mathfrak{I}\left(d \varphi_{t}(W)\right)\right)=\hat{g}(\mathfrak{J}(V), W)$, so

$$
\left(d \varphi_{t}\right) * \mathfrak{J}^{\circ}\left(d \varphi_{t}\right)=\mathfrak{J}
$$

where $\left(d \varphi_{t}\right)^{*}$ is the adjoint operator of $d \varphi_{t}$. A diffeomorphism $F: \mathbb{R}^{2 n} \rightarrow \mathbb{R}^{2 n}$, $F(0)=0$ which preserves a symplectic form is called a symplectic diffeomorphism.

Now, let $\varphi_{t}(\eta)$ be a periodic orbit, and let $S_{\eta} \subset T_{1} M$ be a local transversal section containing $\eta$. Recall that the Poincaré map $\mathfrak{P}_{\eta}: S_{\eta} \rightarrow S_{\eta}$ of the orbit associated to $S_{\eta}$ assigns to each point $q \in S_{\eta}$ the point $\mathfrak{P}_{\eta}(q)=\varphi_{t_{0}}(q)$, where $t_{0}=\inf _{t>0}\left\{\varphi_{t}(q) \cap S_{\eta} \neq \emptyset\right\}$. By the above comments, if $S_{\eta}$ is normal to the direction of the flow at $\eta$, the linear part of the Poincare map is a symplectic isomorphism. Poincaré maps corresponding to different sections containing $\eta$ are conjugate, so we can suppose that there is a unique Poincare map when talking about properties which are invariant under conjugation. Also, up to a change of coordinates we can suppose that $\Omega$ is the canonical form $\omega=\sum_{i=1}^{m} d x_{i} \Lambda d y_{i}$. So let $J_{s}^{k}(2 m)$ be the set of $k$-jets of symplectic automorphisms of $\left(\omega, \mathbb{R}^{2 m}\right)$ which fix $0 \in \mathbb{R}^{2 m}$. Let $Q$ be any subset of $J_{s}^{k}(2 m)$ which is invariant under conjugacies by every $\sigma \in J_{s}^{k}(2 m)$. Then Takens and Klingenberg in [4] show the following theorem:

Theorem 1.1. Let $Q \subset J_{s}^{k}(2 m)$ as above be generic. Then the following property $P_{Q}$ is $C^{k}$-generic in $\kappa^{k}(M):$ the geodesic flow of $g$ has the property $P_{Q}$ if the Poincaré map of every closed orbit belongs to $Q$.

In other words, generic properties of symplectic automorphisms of $\mathbb{R}^{2 n}$ are generic for Poincaré maps of closed orbits of geodesic flows. We now show that:

Proposition 1.1. Let $g \in \operatorname{int}\left(E^{1}(M)\right)$. Then every periodic orbit is hyperbolic.

For the proof we recall a classical result describing local invariant submanifolds of symplectic diffeomorphisms near periodic points [3]:

Lemma 1.1. Let $U \subseteq \mathbb{R}^{2 m}$ be an open neighborhood of $0 \in \mathbb{R}^{2 m}$, and let $P: U \rightarrow U$ be a symplectic diffeomorphism with $P(0)=0$. Let $\bar{P}=d P(0)$ and let

$$
V^{s} \oplus V^{u} \oplus V^{c e}
$$

be the direct splitting of $\mathbb{R}^{2 m}$ into the stable, unstable and central subspaces with respect to $\bar{P}$. Then there exist local imbeddings $W^{s}, W^{u}: \mathbb{R}^{p} \rightarrow \mathbb{R}^{2 m}$ and $W^{c e}: \mathbb{R}^{2 q} \rightarrow \mathbb{R}^{2 m}$ such that $T_{0} W^{s}=V^{s}, T_{0} W^{u}=V^{u}$ and $T_{0} W^{c e}=V^{c e}$. They are called stable, unstable and central manifolds respectively. If $P$ is of class $C^{k}$, these manifolds are of class $C^{k}$ and while $W^{s}$ and $W^{u}$ are unique, $W^{c e}$ is not unique in general.

So for the Poincare map of a closed orbit there exists a central manifold (which could be a point) of the same differentiable class as the map. Let us call $P_{c e}=\left.P\right|_{W^{c e}}$, the restriction of $P$ to $W^{c e}$. 
Definition. $P: \mathbb{R}^{2 m} \rightarrow \mathbb{R}^{2 m}, P(0)=0$ is of twist type if

(a) The corresponding linear map $\bar{P}$ is not hyperbolic.

(b) $\bar{P}$ is elementary (i.e., all the eigenvalues are different).

(c) $\bar{P}$ is 4-elementary (i.e., if $\varrho_{1}, \varrho_{2}, \ldots, \varrho_{k}$ is the set of eigenvalues of $P$ with modulus less than 1 and $\lambda_{k+1}, \ldots, \lambda_{m}$ is any subset of eigenvalues of modulus 1 then for every $m$-tuple $\left(a_{1}, a_{2}, \ldots, a_{m}\right)$ of integers we have

(d) If

$$
\left.\left(\varrho_{1}\right)^{a_{1}} \cdot \ldots \cdot\left(\varrho_{k}\right)^{a_{k}} \cdot\left(\lambda_{k+1}\right)^{a_{k+1}} \cdot \ldots \cdot\left(\lambda_{m}\right)^{a_{m}} \neq 1\right) .
$$

$$
\left(z^{*}\right)^{k}=z^{k} \exp 2 \pi i\left(a^{k}-\sum_{i} b_{i}^{k} z^{i} z^{-i}\right)+w^{k}(z, \bar{z})
$$

is the Birkhoff normal form of $P_{c e}$, then $\operatorname{det}\left(b_{i}^{k}\right) \neq 0$.

Remark that properties (a), (b), (c) are $C^{1}$ generic, and property (d) is $C^{3}$ generic (see for example $[3,8]$ ). As a consequence of Theorem 1.1 we have the following result:

Lemma 1.2. Let $\varphi_{t}(\xi)$ be a periodic orbit of $\varphi_{t}: T_{1} M \rightarrow T_{1} M$ the geodesic flow of $(M, g)$ and let $\gamma(t)=\pi{ }^{\circ} \varphi_{t}(\xi)$ be the underlying geodesic. Let $\mathfrak{P}$ be the Poincaré map. Assume that the linear part $P$ has $2 q$ eigenvalues on the unit circle. Then, in an arbitrarily small tubular neighborhood of $\gamma(t) \subset M$ there exist arbitrarily small perturbations of $g$ supported on these neighborhoods such that, for the perturbed metrics $\gamma(t)$ is still a geodesic, the associated Poincare map is $C^{3}$-close to $\mathfrak{P}$ and its restriction to $W^{c e}(\xi)$ is of twist type.

On the other hand, we have the following generalized version of the socalled Birkhoff-Lewis fixed point theorem, which is due to Moser [8]:

Theorem 1.2. Let $P: \mathbb{R}^{2 n} \rightarrow \mathbb{R}^{2 n}, P(0)=0$ be a locally symplectic diffeomorphism of twist type with no hyperbolic part (i.e., $V^{s}=V^{u}=0$, where $V^{s}$ and $V^{u}$ are given in Lemma 1.1). Then in every neighborhood of 0 there exist infinitely many closed orbits. The number of closed orbits of period $\leqq k$ is finite for every $k \in \mathbb{N}$.

The proof of Proposition 1.1 is as follows: let $g \in \operatorname{int} E^{k}(M)$ and let $\varphi_{t}(\xi)$ be a closed orbit of the geodesic flow. If the Poincare map is not hyperbolic, its linear part has some eigenvalues in the unit sphere. Thus, we can apply Lemma 1.2 to the restriction of the Poincare map to the central manifold $P_{c e}$ and deduce that there exist arbitrarily small perturbations $g_{n}$ of the metric and sequences $\left\{{ }^{n} \varphi_{t}\left(\xi_{m}\right)\right\}$ of closed orbits of ${ }^{n} \varphi_{t}:\left(T_{1} M, g_{n}\right) \rightarrow\left(T_{1} M, g_{n}\right)$ - the geodesic flows of $g_{n}$ - such that $\varphi_{t}(\xi)$ is a closed orbit of ${ }^{n} \varphi_{t}$ for every $n \in \mathbb{N}$ and:

$$
\lim _{m \rightarrow+\infty} \sup _{s \in \mathbb{R}} d_{g_{n}}\left({ }^{n} \varphi_{s}\left(\xi_{m}\right),{ }^{n} \varphi_{s}(\xi)\right)=0 \forall n \in \mathbb{N} .
$$

This means that the metrics $g_{n} \notin E^{1}(M)$ which clearly contradicts the fact that $g \in$ int $E^{1}(M)$.

Following [7] let $\mathscr{F}^{k}(M) \subset \kappa^{k}(M)$ be the set of geodesic flows of Riemannian metrics of $M$ satisfying the following property: for every $\varphi \in \mathscr{F}^{k}(M)$ there exists a neighborhood $V(\varphi) \subset \kappa^{k}(M)$ of $\varphi$ such that if $\psi \in V(\varphi)$ then every closed orbit of $\psi$ is hyperbolic. Then:

Corollary 1.1. int $E^{1}(M) \subset \mathscr{F}^{1}(M)$. 


\section{Invariant Splittings on Persistently Hyperbolic Sets of Periodic Orbits}

Let $\varphi \in \mathscr{F}^{1}(M)$, let $\xi \in T_{1} M$ be a closed orbit. From the last section there exist a stable subspace $E_{\xi}^{s} \subset T_{\xi} T_{1} M$ and an unbstable subspace $E_{\xi}^{u} \subset T_{\xi} T_{1} M$, the former being contracted by $d \varphi_{t}, t \geqq 0$, and the later being contracted by $d \varphi_{t}, t \leqq 0$. Mañe proves in [7] that the bundles $\xi \mapsto E_{\xi}^{s}$ and $\xi \mapsto E_{\xi}^{u}$ defined on $P\left(\varphi_{t}\right)$ admit continuous, $\varphi_{t}$-invariant extensions to bundles on the whole $\overline{P\left(\varphi_{t}\right)}$ with special properties, which are very close to hyperbolicity. For instance, these properties imply the hyperbolicity of the extended bundles [7] in the case of structurally stable diffeomorphisms. This is one of the more difficult, elaborated steps toward the proof of the $C^{1}$-stability conjecture for diffeomorphisms.

Definition. Given a symplectic form $\beta$ in $\mathbb{R}^{2 n}$ a Lagrangian subspace $C$ of $\mathbb{R}^{2 n}$ is defined as:

(a) $\forall V \in X, \beta(V, W)=0 \leftrightarrow W \in X$.

(b) $\operatorname{dim}(X)=n$.

Definition. Let $\varphi_{t}$ be the geodesic flow of $(M, g)$ and let $\Omega_{\xi}$ be the associated symplectic form of $N_{\xi}$ for every $\xi \in T_{1} M$. If $A \cong T_{1} M$ a Lagrangian bundle over $A, \xi \rightarrow L_{\xi}$, is a map which assigns to each $\xi \in A$ a Lagrangian subspace $L_{\xi}$ of $N_{\xi}$. A splitting $S_{\xi} \oplus U_{\xi}=N_{\xi}$ over $A$ is said to be Lagrangian if both $\xi \rightarrow S_{\xi}$ and $\xi \rightarrow U_{\xi}$ are Lagrangian bundles over $A$.

Let us make precise the extension theorem [7]:

Theorem 2.1. If $\varphi_{t} \in \operatorname{int}\left(E^{1}(M)\right)$ there exist a neighborhood $U$ of $\varphi_{t}$ in $\operatorname{int}\left(E^{1}(M)\right)$ and constants $K>0, D>0,0<\lambda<1$ such that:

(a) If $\varphi_{t}^{\prime}:\left(T_{1} M, g^{\prime}\right) \rightarrow\left(T_{1} M, g^{\prime}\right)$ belongs to $U$ and $\varphi_{t}^{\prime}(\zeta) \in P\left(\varphi_{t}^{\prime}\right)$ has minimum period $w \geqq D$, then

$$
\prod_{i=0}^{k-1}\left\|\left.d \varphi_{D}^{\prime}\right|_{E_{\phi_{i D}(5)}^{\prime s}}\right\|_{g^{\prime}} \leqq K \lambda^{k}
$$

and

$$
\prod_{i=0}^{k-1}\left\|\left.d \varphi_{-i D}^{\prime}\right|_{E_{\phi_{-i D}^{\prime}(\xi)}^{\prime u}}\right\| \|_{g^{\prime}} \leqq K \lambda^{k}
$$

where $E_{\xi}^{\prime s} \oplus E_{\xi}^{\prime u} \oplus E_{\xi}^{\prime}=T_{\xi}\left(T_{1} M\right)$ is the hyperbolic splitting for $\xi$ in the orbit $\varphi_{t}^{\prime}(\zeta)$, and $k=\left[\frac{w}{D}\right]$.

(b) There exists a continuous splitting for $T_{\xi}\left(T_{1} M\right)=G_{\xi}^{s} \oplus G_{\xi}^{u} \oplus E_{\xi}, \xi \in \overline{P\left(\varphi_{t}\right)}$ with

$$
\left\|\left.\left(d_{\xi} \varphi_{D}\right)\right|_{G_{\xi}^{s}}\right\| \circ\left\|\left.\left(d_{\varphi_{D}(\xi)} \varphi_{-D}\right)\right|_{G_{\phi_{D}(\xi)}^{u}}\right\| \leqq \lambda
$$

and $G_{\xi}^{s}=E_{\xi}^{s}, G_{\xi}^{u}=E_{\xi}^{u}$ if $\xi \in P\left(\varphi_{t}\right)$.

If $\psi_{t}: \Sigma \rightarrow \Sigma$ is a differentiable flow acting on a manifold $\Sigma$, and $X$ is an invariant subset of $\Sigma$ in which the flow has no singularities, an invariant splitting $S_{\xi} \oplus U_{\xi} \oplus E_{\xi}=T_{\xi} \Sigma$ defined on every $\xi \in X$ is said to be dominated if there exist constants $0<\delta<1, m>0$ such that $\left\|\left.d \psi_{m}\right|_{s_{\xi}}\right\| \cdot\left\|\left.d \psi_{-m}\right|_{U_{\psi_{m}(\xi)}}\right\| \leqq \delta$. The space $E_{\xi}$ is as before the direction of the flow in $\xi$. Statement (b) in the last 
theorem says that $G_{\xi}^{s} \oplus G_{\xi}^{u} \oplus E_{\xi}=T_{\xi} T_{1} M, \xi \in \overline{P(\varphi)}$ is dominated. Hyperbolic splittings are clearly dominated. The converse of this assertion is not true in general. However, we shall prove that in the case of Lagrangian splittings the domination condition is equivalent to hyperbolicity.

Lemma 2.1. $\xi \mapsto G_{\xi}^{s}$ is Lagrangian for $\xi \in \overline{P\left(\varphi_{t}\right)}$.

Proof. Since the bundle is continuous on $\overline{P\left(\varphi_{t}\right)}$ it suffices to show that $\xi \mapsto G_{\xi}^{s}$ is Lagrangian for $\xi \in P\left(\varphi_{t}\right)$. So let $\varphi_{t}(\xi)$ be a periodic orbit and $T_{\varphi_{t}(\xi)}\left(T_{1} M\right)$ $=G_{\varphi_{t}(\xi)}^{s} \oplus G_{\varphi_{t}(\xi)}^{u} \oplus E_{\varphi_{t}(\xi)}$ be the corresponding splitting. Let $T>0$ be the minimum period of $\xi$. Recall that there exist $K(\xi)>0,0<\lambda(\xi)<1$ such that if $V \in G_{\xi}^{s}=E_{\xi}^{s}$ then

$$
\left\|d \varphi_{t}(V)\right\| \leqq K(\xi) \lambda(\xi)^{t}\|V\| \forall t \geqq 0 .
$$

First of all, $E_{\xi}^{s}$ and $E_{\xi}^{u}$ are perpendicular to $E_{\xi} \forall \xi \in \overline{P(\varphi)}$ : indeed, let us suppose that $V \in E_{\xi}^{s}$ is written as $V=\alpha+\beta$, where $\alpha \in N_{\xi}, \beta \in E_{\xi}$. Since $d \varphi_{t}$ preserves $N_{\xi}$ and $E_{\xi}$, and $\left\|d \varphi_{t}(W)\right\|=\|W\|$ for every $W \in E_{\xi}$ we have

$$
\begin{aligned}
\left\|d \varphi_{t}(V)\right\|^{2} & =\left\|d \varphi_{t}(\alpha)\right\|^{2}+\left\|d \varphi_{t}(\beta)\right\|^{2} \\
& \geqq\left\|d \varphi_{t}(\beta)\right\|^{2} \\
& =\|\beta\|^{2}, \\
\rightarrow \quad\|\beta\| & \leqq \lim _{t \rightarrow+\infty}\left\|d \varphi_{t}(V)\right\|=0 .
\end{aligned}
$$

So $E_{\xi}^{s} \subset N_{\xi}$. Similarly, $E_{\xi}^{u} \subset N_{\xi}$.

Since $\mathfrak{J}$ is an isometry we have

$$
\left\|d \varphi_{t}(V)\right\|=\left\|\mathfrak{J}^{\circ} d \varphi_{t}(V)\right\| .
$$

Hence if $V, W \in E_{\xi}^{s}$ we have

$$
\begin{aligned}
|\Omega(V, W)| & =\left|\Omega\left(d \varphi_{t}(V), d \varphi_{t}(W)\right)\right|=\left|\hat{g}\left(d \varphi_{t}(V), \mathfrak{J}^{\circ} d \varphi_{t}(W)\right)\right| \\
& \leqq K(\xi)^{2} \lambda(\xi)^{-2 t}\|V\| \circ\|W\| \forall t \geqq 0 \\
\rightarrow \quad|\Omega(V, W)| & =\lim _{t \rightarrow+\infty} K(\xi)^{2} \lambda(\xi)^{-2 t}\|V\| \circ\|W\|=0 .
\end{aligned}
$$

Similarly, $\Omega(V, W)=0 \forall V, W \in E_{\xi}^{u}$. Now, recall the following property of Lagrangian subspaces:

Sublemma. A subspace $X$ of $\left(\mathbb{R}^{2 n}, \omega\right)$ is Lagrangian if and only if

i) $\omega(Z, V)=0 \forall Z, V \in X$.

ii) there exists a subspace $Y$ of $\mathbb{R}^{2 n}$ such that $X \oplus Y=\mathbb{R}^{2 n}$ and $\omega(V, W)$ $=0 \forall V, W \in Y$.

Therefore, Lemma 2.1 holds from the sublemma applied to $X=E_{\xi}^{s}, Y=E_{\xi}^{u}$ and $\left(N_{\xi}, \Omega_{\xi}\right)$.

Proposition 2.1. Let $S_{\xi} \oplus U_{\xi}=N_{\xi}$ be a continuous, invariant Lagrangian splitting defined on a compact, invariant set $X \cong T_{1} M$. The splitting is dominated if and only if it is hyperbolic, where $S_{\xi}$ is forward-contracted by $\varphi_{t}$ and $U_{\xi}$ is backward-contracted by $\varphi_{t}$.

We prove first two lemmas. 
Lemma 2.2. Let $S_{\xi} \oplus U_{\xi}=N_{\xi}$ be a continuous Lagrangian splitting defined on a compact invariant set $X \cong T_{1} M$. Then $\lim _{k \rightarrow+\infty}\left\|\left(d_{\xi} \varphi_{m}\right)^{k}(\beta)\right\|=+\infty \forall \beta \in U_{\xi}$, $\forall \xi \in X$ if and only if $\lim _{k \rightarrow+\infty}\left\|\left.\left(d_{\xi} \varphi_{m}\right)^{k}\right|_{S_{\xi}}\right\|=0$. Similarly, $S_{\xi}$ is backward-expanded by $\varphi_{m}$ if and only if $U_{\xi}$ is backward-contracted.

Proof. $(\Rightarrow)$ Consider the family of linear operators

$$
\left\{T_{v}: U_{\xi} \rightarrow \mathbb{R}, v \in S_{\xi},\|v\|=1, \xi \in X\right\}
$$

defined by $T_{v}(w)=\Omega(v, w)$. Since the splitting $S_{\xi} \oplus U_{\xi}=N_{\xi}$ is both continuous and Lagrangian and $X$ is a compact set this is a compact family of linear, non-trivial operators. So for every $\xi \in X$ and $v \in S_{\xi}$ the kernel $K(v)$ of $T_{v}$ is a codimension 1 subspace of $U_{\xi}$ and there exists $w=w(v) \in U_{\xi}$ such that

$$
T_{v}(w)=1
$$

and

$$
g(K(v), w)=0 .
$$

This dual vector $w=w(v)$ depends continuously on $\xi \in X$ and $v \in S_{\xi}$ and there exist constants $0<C_{1} \leqq C_{2}$ such that

$$
C_{1} \leqq\|w(v)\| \leqq C_{2}
$$

for every pair $(\xi, v) \in S_{0}=\left\{(\xi, v), \xi \in X, v \in S_{\xi},\|v\|=1\right\}$. Remark also that $d \varphi_{t}(K(v))=K\left(d \varphi_{t}(v)\right)=K\left(\frac{d \varphi_{t}(v)}{\left\|d \varphi_{t}(v)\right\|}\right)$ because $\Omega$ is invariant by the flow $\varphi_{t}$. So take a vector $(\xi, v) \in S_{0}$ and consider $w=w(v) \in U_{\xi}$. From the hypotheses we get that

$$
\lim _{n \rightarrow+\infty}\left\|d \varphi_{m}^{n}(w)\right\|=+\infty,
$$

and from the compactness of $S_{0}$ the vectors $w(v)$ are uniformly expanded by the positive iterates of $\varphi_{m}$. Then we have

$$
\begin{aligned}
1=\Omega(v, w) & =T_{v}(w) \\
& =\Omega\left(d \varphi_{m}^{n}(v), d \varphi_{m}^{n}(w)\right) \\
& =\Omega\left(\frac{d \varphi_{m}^{n}(v)}{\left\|d \varphi_{m}^{n}(v)\right\|},\left\|d \varphi_{m}^{n}(v)\right\| \cdot d \varphi_{m}^{n}(w)\right) \\
& =T_{v_{n}}\left(\left\|d \varphi_{m}^{n}(v)\right\| \cdot d \varphi_{m}^{n}(w)\right),
\end{aligned}
$$

where $v_{n}=\frac{d \varphi_{m}^{n}(v)}{\left\|d \varphi_{m}^{n}(v)\right\|}$. Obviously $\left(\varphi_{m}^{n}(\xi), v_{n}\right) \in S_{0} \forall n$, and from the last equality we deduce that there exists a vector $z_{n} \in K\left(v_{n}\right)$ such that

$$
\left\|d \varphi_{m}^{n}(v)\right\| \cdot d \varphi_{m}^{n}(w)=w\left(v_{n}\right)+z_{n} .
$$

Since $K(\alpha)$ is $\varphi_{t}$-invariant $\forall t$ there exists $k_{n} \in K(v)$ such that $d \varphi_{m}^{n}\left(k_{n}\right)=z_{n}$. This implies that

$$
\begin{aligned}
w\left(v_{n}\right) & =\left\|d \varphi_{m}^{k(n)}(v)\right\| \cdot d \varphi_{m}^{k(n)}(w)-d \varphi_{m}^{k(n)}\left(k_{n}\right) \\
& =d \varphi_{m}^{k(n)}\left(\left\|d \varphi_{m}^{k(n)}(v)\right\| \cdot w-k_{n}\right) .
\end{aligned}
$$


The vectors $\left\|d \varphi_{m}^{k(n)}(v)\right\| \cdot w-k_{n}$ belong to $U_{\xi}$, so by the hypotheses they must be expanded by the positive iterates of $\varphi_{m}$. But since $C_{1} \leqq\left\|w\left(v_{n}\right)\right\|$ $\leqq C_{2}$, this means that

$$
\|\| d \varphi_{m}^{k(n)}(v)\left\|\cdot w-k_{n}\right\| \rightarrow 0
$$

if $n$ goes to $+\infty$. Since $w=w(v)$ and $K(v)$ are perpendicular this implies that

$$
\lim _{n \rightarrow+\infty}\left\|d \varphi_{m}^{k(n)}(v)\right\| \cdot w=0
$$

and therefore $\lim _{n \rightarrow+\infty}\left\|d \varphi_{m}^{k(n)}(v)\right\|=0$ which proves the assertion.

$(\Leftarrow)$ If there exist $w \in U_{\xi}$, a sequence $k(n) \rightarrow+\infty$ and $D>0$ such that $\left\|d \varphi_{m}^{k(n)}(w)\right\| \leqq D$ for every $k \geqq 0$, then for every $v \in S_{\xi}$ we have

$$
\begin{aligned}
|\Omega(v, w)| & =\left|\Omega\left(d \varphi_{m}^{k(n)}(v), d \varphi_{m}^{k(n)}(w)\right)\right| \\
& \leqq\left\|d \varphi_{m}^{k(n)}(v)\right\| \cdot\left\|d \varphi_{m}^{k(n)}(w)\right\| \\
& \leqq D \cdot\left\|d \varphi_{m}^{k(n)}(v)\right\| \rightarrow 0
\end{aligned}
$$

by hypotheses. This implies that $\Omega(v, w)=0 \forall v \in S_{\xi}$. But since $S_{\xi}$ is Lagrangian this means that $w \in S_{\xi} \cap U_{\xi}=\{0\}$. The proof of the second statement of Lemma 2.2 is completely analogous.

Lemma 2.3. Let $S_{\xi} \oplus U_{\xi}=N_{\xi}$ be a continuous, dominated Lagrangian splitting defined on a compact, invariant set $X \subseteq T_{1} M$. Then $\lim _{k \rightarrow+\infty}\left\|d \varphi_{m}^{k}(w)\right\|=+\infty$ $\forall w \in U_{\xi}, \forall \xi \in X$. In a similar way, $\lim _{k \rightarrow+\infty}\left\|d \varphi_{m}^{k}(v)\right\|=+\infty \forall v \in S_{\xi}, \forall \xi \in X$. Here, $m>0$ is the constant appearing in the domination condition.

Proof. If $\lim _{k \rightarrow+\infty}\left\|d \varphi_{m}^{k}(v)\right\|=0 \forall v \in S_{\xi}$ the statement is a consequence of Lemma 2.2. So let us suppose that there exist $\xi \in X, v \in S_{\xi}, \delta>0$ and a sequence $k(n) \rightarrow+\infty$ such that $\left\|d \varphi_{m}^{k(n)}(v)\right\| \geqq \delta$. Without loss of generality we can take $\delta=1$. From the domination condition we get

$$
\left\|\left.d \varphi_{m}^{k(n)}\right|_{S_{\xi}}\right\| \cdot\left\|\left.d \varphi_{m}^{-k(n)}\right|_{U_{\xi}}\right\| \leqq \prod_{i=1}^{k(n)-1}\left\|\left.d \varphi_{m}\right|_{S_{\phi_{m}^{i}(\xi)}}\right\| \cdot\left\|\left.d \varphi_{m}^{-1}\right|_{U_{\phi_{m}^{i+1}(\xi)}}\right\| \leqq \lambda^{k(n)-1}
$$

where $\lambda \in(0,1)$ gives the domination in the splitting. On the other hand, if $A: \mathbb{R}^{n} \rightarrow \mathbb{R}^{n}$ is an invertible linear map, we have that $\|v\|=\left\|A^{-1} \circ A(v)\right\|$

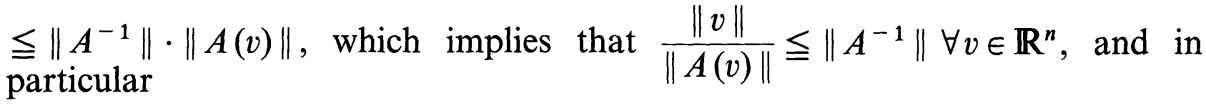

$$
\frac{1}{\inf _{\|v\|=1}\|A(v)\|} \leqq\left\|A^{-1}\right\|
$$

The contradiction assumption and the last two inequalities imply

$$
\begin{aligned}
& \frac{1}{\inf _{\|v\|=1}\left\|\left.d \varphi_{m k(n)}\right|_{U_{\xi}}\right\|} \leqq\left\|\left.d \varphi_{m}^{k(n)}\right|_{S_{\xi}}\right\| \cdot\left\|\left.d \varphi_{m}^{-k(n)}\right|_{U_{\xi}}\right\| \leqq \lambda^{k(n)-1} \\
\Rightarrow \quad & \inf _{\|v\|=1}\left\|\left.d \varphi_{m}^{k(n)}\right|_{U_{\xi}}\right\| \geqq(1 / \lambda)^{-(k(n)-1)} \rightarrow+\infty .
\end{aligned}
$$


Now, from the compactness of $X$ and the continuity of the bundle $\xi \rightarrow U_{\xi}$ it is easy to check that

$$
\lim _{k \rightarrow+\infty}\left\|d \varphi_{m}^{k}(w)\right\|=+\infty
$$

for every $w \in U_{\xi}$, and every $\xi \in X$. Analogously we can prove that $\lim _{k \rightarrow+\infty}\left\|d \varphi_{m}^{k}(v)\right\|=+\infty \forall v \in S_{\xi}, \forall \xi \in X$.

Proof of Proposition 2.1. From Lemmas 2.2 and 2.3 we have that

$$
\begin{aligned}
& \lim _{k \rightarrow+\infty}\left\|d \varphi_{m}^{k}(v)\right\|=0 \forall v \in S_{\xi}, \quad \forall \xi \in X, \\
& \lim _{k \rightarrow-\infty}\left\|d \varphi_{m}^{k}(w)\right\|=0 \forall w \in U_{\xi}, \quad \forall \xi \in X .
\end{aligned}
$$

Now, recall the following lemma due to Eberlein [2]:

Lemma. Let $f: \mathbb{R}^{+} \rightarrow \mathbb{R}^{+}$be a continuous function satisfying

i) $\lim _{t \rightarrow+\infty} f(t)=0$.

ii) $f(t+s) \leqq f(t) \cdot f(s)$.

Then there exist $L>0,0<a<1$ such that $f(t) \leqq L a^{t}$.

We can apply this lemma to the function $f_{\xi}(t)=\left\|\left.d \varphi_{t}\right|_{s_{\xi}}\right\|$ for each $\xi \in X$ and deduce that there are constants $L(\xi)>0,0<a(\xi)<1$ such that $\left\|\left.d \varphi_{t}\right|_{s_{\xi}}\right\| \leqq L(\xi) a(\xi)^{t} \forall t \geqq 0$. From the compactness of $X$ and the continuity of the bundle $S_{\xi}$ it is straightforward to deduce that there exist $E>0$ and $0<\alpha<1$ such that

$$
\left\|\left.d \varphi_{t}\right|_{s_{\xi}}\right\| \leqq E \alpha^{t} \forall t \geqq 0, \forall \xi \in X .
$$

By the same reasoning we get constants $D>0,0<v<1$ such that

$$
\left\|\left.d \varphi_{-t}\right|_{U_{\xi}}\right\| \leqq D v^{t} \forall t \geqq 0, \forall \xi \in X
$$

Taking $C=\sup (E, D)$ and $\lambda=\sup (\alpha, v)$ we have that the set $X$ is a hyperbolic set for $\varphi_{t}$ with splitting $S_{\xi} \oplus U_{\xi}=T_{\xi} M$ and constants $C$ and $\lambda$. This concludes the proof of the proposition.

\section{Density of Periodic Orbits of Expansive Geodesic Flows}

We shall expose first some canonical facts of the theory of expansive systems. Our main references are $[4,10]$. Throughout this section $N$ will be a compact manifold.

Definition. Let $\psi_{t}: N \rightarrow N$ be a continuous flow acting on $N$. For a given $\varepsilon>0$, let $\widetilde{C}_{\varepsilon}^{s}(p)$ be the set of points $q$ of $N$ with the following property: There exists a continuous, surjective $\operatorname{map} \alpha: \mathbb{R}^{+} \rightarrow \mathbb{R}^{+}$with $\alpha(0)=0$ such that

$$
d\left(\psi_{t}(p), \psi_{\alpha(t)}(q)\right) \leqq \varepsilon
$$

for every $t \geqq 0$. Analogously, let $\tilde{C}_{\varepsilon}^{u}(p)$ be the set of points $q$ in $N$ such that there exists a continuous, surjective function $\beta: \mathbb{R}^{-} \rightarrow \mathbb{R}^{-}$with $\beta(0)=0$ 
such that

$$
d\left(\psi_{t}(p), \psi_{\beta(t)}(q)\right) \leqq \varepsilon
$$

for every $t \in \mathbb{R}^{-}$.

Notice that $\tilde{C}_{\varepsilon}^{s}(p)$ is forward invariant by $\psi_{t}$ in the following sense: for every $t \geqq 0$ and $q \in \widetilde{C}_{\varepsilon}^{s}(p)$ there exists $t_{0} \geqq 0$ such that $\psi_{t_{0}}(q) \in \widetilde{C}_{\varepsilon}^{s}\left(\psi_{t}(p)\right)$. If the flow $\psi_{t}$ were an Anosov flow the set $\widetilde{C}_{\varepsilon}^{s}(p)$ would be the result of intersecting the weak stable submanifold of $p$ (i.e. the saturated stable submanifold of $p$ ) with some neighborhood of $p$ and then taking the connected component of this set containing $p$. Similarly, for every $t \leqq 0$ and $q \in \tilde{C}_{\varepsilon}^{u}(p)$ there exists $t_{1} \leqq 0$ such that $\psi_{t_{1}}(q) \in \tilde{C}_{\varepsilon}^{u}\left(\psi_{t}(p)\right)$.

Theorem 3.1. Let $\operatorname{dim} N=3$ and let $\psi_{t}: N \rightarrow N$ be an expansive flow without singularities with expansivity constant $\varepsilon>0$. Then the following assertions are true:

a) There exists $\delta>0$ such that $\tilde{C}_{\delta}^{s}(\xi), \tilde{C}_{\delta}^{u}(\xi)$ are connected, non-trivial sets $\forall \xi \in N$ (i.e., they do contain points which are not in the orbit of $\xi$ ).

b) Let $\Sigma_{\xi}=\exp _{\xi}\left\{w \in T_{\xi} N \backslash\|w\|\langle\varepsilon,\langle w, X(\xi)\rangle=0\}\right.$, where $\langle$, $\rangle$ is the metric of $N$. Then there exist periodic points $\eta_{i}, i=1,2, \ldots, n$ such that for every point $\xi \in G=N-\bigcup_{i, t \in \mathbb{R}}\left\{\psi_{t}\left(\eta_{i}\right)\right\}$ there exists an open neighborhood $V$ of $\xi$ with diameter less than $\frac{\varepsilon}{2}$ such that the sets

$$
\begin{aligned}
& C_{\delta}^{s}(\zeta)=\Sigma \cap V \cap \tilde{C}_{\delta}^{s}(\zeta), \\
& C_{\delta}^{u}(\zeta)=\Sigma \cap V \cap \widetilde{C}_{\delta}^{u}(\zeta)
\end{aligned}
$$

are connected curves satisfying a local product structure: There exists a homeomorphism

$$
F:(0,1)^{2} \rightarrow V \cap \Sigma
$$

such that

(i) $F(0,0)=\zeta$,

(ii) $F_{x}(z)=C_{\delta}^{s}(F(x, z))$,

(iii) $F^{z}(x)=C_{\delta}^{u}(F(x, z))$,

for every $x, z$ in $(0,1)$, where $F_{x}:(0,1) \rightarrow \Sigma, F^{z}:(0,1) \rightarrow \Sigma$ are the maps $F_{x}(z)$ $=F(x, z), F^{z}(x)=F(x, z)$.

(c) $\tilde{C}_{\delta}^{s}(\xi)$ contracts uniformly as $t$ goes to $+\infty$ (respectively $\tilde{C}_{\delta}^{u}(\xi)$ ) contracts with $t \rightarrow-\infty)$. In other words, for every $t>0, v>0$ there exist $T>0$ such that

$$
\psi_{r}\left(\tilde{C}_{\delta}^{s}(\xi)\right) \subset \bigcup_{s \leqq t} \tilde{C}_{\alpha}^{s}\left(\psi_{t}(\xi)\right)
$$

for every $t \geqq T$ and every $\xi \in G$.

In particular, $C_{\delta}^{s}(\xi), C_{\delta}^{u}(\xi)$ are connected curves with intersect only at $\xi$, and they depend continuously on $\xi \in G$.

Recall that a point $p$ is non-wandering for a flow $\psi_{t}: N \rightarrow N$ if for every open neighborhood $V$ of $p$ there exists a sequence $\left\{t_{n}\right\}$ of real numbers with $\left|t_{n}\right| \rightarrow+\infty$ and $\psi_{t_{n}}(V) \cap V \neq \emptyset$. 
Proposition 3.1. Let $\psi_{t}: N \rightarrow N$ be expansive in $N$, where $\operatorname{dim} N=3$. If the set of non-wandering points of $\psi_{t}$ is dense in $N$ then the set of periodic orbits of $\psi_{t}$ is also dense.

Proof. Let $\xi \in G, V(\xi)$ and $\Sigma$ as in Theorem 3.1. From Theorem 3.1(b) there exist projections

$$
\begin{aligned}
& \Pi_{s}: \Sigma \cap V \rightarrow C_{\delta}^{s}(\xi), \\
& \Pi_{u}: \Sigma \cap V \rightarrow C_{\delta}^{u}(\xi),
\end{aligned}
$$

defined by $\Pi_{s}(\zeta)=C_{\delta}^{s}(\xi) \cap C_{\delta}^{u}(\zeta), \Pi_{u}(\zeta)=C_{\delta}^{u}(\xi) \cap C_{\delta}^{s}(\zeta)$ for every $\zeta \in \Sigma \cap V$. Remark that by Theorem 3.1(b), $F\left[(0,1)^{2}\right]=V \cap \Sigma$ is homeomorphic to $C_{\delta}^{s}(\xi) \times C_{\delta}^{u}(\xi)$ : for every $\zeta \in V \cap \Sigma$ there are well-defined coordinates

$$
\zeta \rightarrow\left(\Pi_{s}(\zeta), \Pi_{u}(\zeta)\right)
$$

Suppose that $\xi$ is a non-wandering point. Let $P: \Sigma \cap V \rightarrow \Sigma \cap V$ be the Poincare map of the flow $\psi_{t}$. There are sequences $\left\{\xi_{n}\right\}$ of points in $\Sigma$ such that $\xi_{n} \rightarrow \xi$, and $\left\{k_{n}\right\}$ of integers with $\left|k_{n}\right| \rightarrow+\infty$ such that $P^{k_{n}}\left(\xi_{n}\right) \rightarrow \xi$. We can suppose that $k_{n} \geqq 0 \forall n$ without lost of generality. They correspond to sequences $\xi_{n} \rightarrow \xi$ of points and $t_{n} \rightarrow+\infty$ such that $\psi_{t_{n}}\left(\xi_{n}\right)=P^{k_{n}}\left(\xi_{n}\right)$. Consider an open neighborhood $V_{\alpha}$ of radius $\alpha$ of $\xi$ with $\bar{V}_{\alpha} \subset V$. From Theorem 3.1 (c) we can deduce that there exist $Q>0$ such that

(i) $d\left(P^{k_{n}}\left(\xi_{n}\right), \xi\right)<\frac{\alpha}{4} \forall n \geqq Q$,

(ii) $d\left(P^{k_{n}}\left(C_{\delta}^{s}\left(\xi_{n}\right)\right), P^{k_{n}}\left(\xi_{n}\right)\right)<\frac{\alpha}{4} \forall n \geqq Q$,

(iii) $C_{\delta}^{u}(\zeta) \subset P^{k_{n}}\left(C_{\delta}^{u}(\zeta)\right) \forall n \geqq Q, \forall \zeta$ not belonging to the orbit of any $\eta_{i}$ (Theorem 3.1(a)). From (i) and (ii) we get that

$$
\begin{aligned}
d\left(P^{k_{n}}\left(C_{\delta}^{s}\left(\xi_{n}\right)\right), \xi\right) & <\frac{\alpha}{2}, \\
P^{k_{n}}\left(C_{\delta}^{s}\left(\xi_{n}\right)\right) & \subset V_{\alpha}
\end{aligned}
$$

for every $n \geqq Q$. Let us consider the restriction $\hat{\Pi}_{s}$ of $\Pi_{s}$ to $C_{\delta}^{s}\left(\xi_{n}\right)$. From this last statement and (iii) the map $\Pi_{s}$ is well defined in the set $P^{k_{n}}\left(C_{\delta}^{s}\left(\xi_{n}\right)\right)=P^{k_{n}}\left(\hat{\Pi}_{s}^{-1}\left(C_{\delta}^{s}(\xi)\right)\right)$ and

$$
\Pi_{s}\left(P^{k_{n}}\left(C_{\delta}^{s}\left(\xi_{n}\right)\right)\right) \subset C_{\delta}^{s}(\xi) .
$$

This implies that the map $\Pi_{s} \circ P^{k_{n}} \circ \hat{\Pi}_{s}^{-1}: C_{\delta}^{s}(\xi) \rightarrow C_{\delta}^{s}(\xi)$ has a fixed point $\xi_{0}$ that, according to the construction, satisfies

$$
\begin{aligned}
\xi_{0} & \subset C_{\delta}^{u}\left(P^{k_{n}}\left(\xi_{0}\right)\right) \\
\rightarrow \quad C_{\delta}^{u}\left(\xi_{0}\right)=C_{\delta}^{u}\left(P^{k_{n}}\left(\xi_{0}\right)\right) & \subset P^{k_{n}}\left(C_{\delta}^{u}\left(\xi_{0}\right)\right)
\end{aligned}
$$

by (iii). But this means that the map

$$
P^{-k_{n}}: C_{\delta}^{u}\left(\xi_{0}\right) \rightarrow C_{\delta}^{u}\left(\xi_{0}\right)
$$

has a fixed point $\xi_{1}$. Since $\alpha$ is arbitrary, and non-wandering points are dense, the periodic orbits are dense as well 
Corollary 3.1. If the geodesic flow of a compact surface is expansive, then the closed orbits are dense.

This is due to the fact that every point of $T_{1} M$ is non-wandering, so Proposition 3.1 applies with $N=T_{1} M$ and $\psi_{t}=\varphi_{t}$.

Acknowledgements. This paper contains part of my thesis work held at IMPA. I am specially grateful to Prof. Ricardo Mañé for his various remarks on the subject, and to Prof. Jorge Lewowicz to whom I one the main ideas of the proof of Proposition 3.1.

\section{References}

1. Anosov, D.: Geodesic flow on closed Riemannian manifolds of negative curvature. Trudy Math. Inst. Steklov 90, (1967)

2. Eberlein, P.: When is a geodesic flow of Anosov type I. J. Diff. Geom. 8, 437-463 (1973)

3. Klingenberg, W.: Lectures on closed geodesics. Berlin, Heidelberg, New York: Springer 1978

4. Klingenberg, W., Takens, F.: Generic properties of geodesic flows. Math. Ann. 197, $323-$ $334(1972)$

5. Lewowicz, J.: Expansive homeomorphisms of surface. Preprint

6. Mañe, R.: Quasi-Anosov diffeomorphisms. Lecture Notes in Math. Vol. 468, pp. 27-29. Berlin, Heidelberg, New York: Springer 1974

7. Mañe, R.: An ergodic closing lemma. Ann. Math. 116, 503-540 (1982)

8. Moser, J.: Proof of a generalised form of a fixed point theorem due to G. D. Birkhoff. Lecture Notes in Math. Vol. 597, pp. 464-549. Berlin, Heidelberg, New York: Springer 1977

9. Pugh, C.: An improved closing lemma and a general density theorem. Am. J. Math. 89, 1010-1021 (1967)

10. Pugh, C., Shub, M.: The $\Omega$-stability theorem for flows. Invent. Math. 11, 150-158 (1970)

11. Paternain, M.: Expansive flows and the fundamental group. Tese de Doutorado. IMPA (1990)

12. Smale, S.: The $\Omega$-stability theorem. Proc. Symp. Pure Math. 14 (1970). Am. Math. Soc., pp. $289-298$

Communicated by J.-P. Eckmann 
